\title{
Percutaneous Nerve Evaluation Test Versus Staged Test Trials for Sacral Neuromodulation: Sensitivity, Specificity, and Predictive Values of Each Technique
}

\author{
Mai Banakhar', Magdy Hassouna ${ }^{2}$ \\ ${ }^{1}$ King Abdulaziz University, Faculty of Medicine, Jeddah, Saudi Arabia \\ ${ }^{2}$ University of Toronto, University Health Network, Toronto Western Hospital, Toronto, Canada
}

Purpose: InterStim device is an U.S. Food and Drug Administration approved minimal invasive therapy for sacral neuromodulation for lower urinary tract dysfunction. Before InterStim implantation, a trial with the appropriate screening tests is required to determine patient therapy eligibility. There are two different techniques for patient screening: percutaneous nerve evaluation (PNE) test and staged test. Few studies have reported success and failure rates for each technique. However, test sensitivity and predictive values of either test have not been studied. The aim of our study was to determine the sensitivity and specificity of each test and to establish a decision algorithm for the most appropriate testing method to be used as a screening test.

Methods: This cross-sectional study was conducted from August 2009 to February 2012 and included patients with lower urinary tract dysfunction who participated in the stimulation test trial. Patients underwent PNE as the first stimulation test, while those who encountered technical difficulty during PNE or electrode migration underwent staged testing.

Results: A total of 213 patients, including 172 female and 41 male subjects, underwent PNE. The patients' diagnoses included refractory overactive bladder (47.9\%), nonobstructive urinary retention (29.6\%), and frequency urgency syndrome (22.1\%). A total of 202 patients were screened with PNE and 10 patients with staged testing. Overall sensitivity of PNE was $87.3 \%$, and it was $90 \%$ for staged test. PNE specificity was $98.5 \%$ as compared to $92.9 \%$ for staged test. Positive and negative predictive values for PNE were $99 \%$ and $82.1 \%$ and for staged test were $90 \%$ and $92.9 \%$, respectively.

Conclusions: PNE test has high specificity and positive predictive value. We recommend PNE, a simple office-based, less expensive procedure as the first option for screening.

Keywords: Sacral Nerve Stimulation; Sacral Neuromodulation; Screening; Staged; Percutaneous Nerve Evaluation Test

- Research Ethics: This study was approved by University Health Network Research Ethics Board (10-0449-AE).

- Conflict of Interest: No potential conflict of interest relevant to this article was reported.

\section{INTRODUCTION}

InterStim device (Medtronic Inc., Minneapolis, MN, USA) is an U.S. Food and Drug Administration approved therapy for sacral nerve stimulation for refractory overactive bladder $(\mathrm{OAB})$, frequency-urgency syndrome and nonobstructive urinary retention [1-5]. Patients' eligibility for sacral neuromodulation (SNM) therapy is determined by their response to an ex-

Corresponding author: Mai Banakhar (D) http://orcid.org/0000-0002-2341-1270 Department of Urology, King Abdulaziz University, Faculty of Medicine, Jeddah, Saudi Arabia

E-mail: drmaibanakher@hotmail.com / Tel: +966-593435049 / Fax: +966-126408347

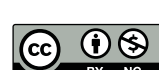

This is an Open Access article distributed under the terms of the Creative Commons Attribution Non-Commercial License (http://creativecommons.org/licenses/by-nc/4.0/) which permits unrestricted non-commercial use, distribution, and reproduction in any medium, provided the original work is properly cited. 
ternal stimulator during the test period. Accurate screening test is crucial in patient selection for SNM and for optimal results. There are 2 different screening methods: percutaneous nerve evaluations (PNE) test and staged test. Patients who show more than $50 \%$ reduction in urinary symptoms during any of the tests are considered as good candidates for definitive SNM therapy $[1,3]$.

PNE testing is done under local anesthesia; patients carry a temporary electrode wire for few days ( $3-5$ days), which is removed in clinic. PNE test has 50\% rate of false negative results due to electrode migration that can be detected subjectively by changing in the type and location of stimulation and objectively by using radiography. However, staged test trial is performed in an operating room, by definitive implantation of a tined electrode (3889, Medtronic Inc.) that minimizes the risk of electrode migration. Patient is allowed to undergo the test for a period of 14 days, and this might increase the risk of wound infection. Staged test trial requires 2 surgeries and incurs more expenses if the trial is unsuccessful, since the tined electrode is removed surgically in the operating room [6].

Upon reviewing the literature, some studies report differences in the implantation rate and conclude that staged test has a better outcome than PNE in selecting candidates for SNM $[7,8]$. Other studies concluded that PNE test is better when comparing reoperation rate using sensory response assessment [9]. The salient point is that the literature regarding SNM lacks the description of a decision algorithm, which is important for screening patients for SNM therapy.

The aim of our study was to assess the sensitivity and the specificity of each method, to establish a decision algorithm for patient selection, and to evaluate any correlation between sensitivity, specificity with patient demographics.

\section{MATERIALS AND METHODS}

We conducted a cross-sectional study between August 2009 and February 2012, after obtaining approval from University Health Network Research Ethics Board (10-0449-AE). The study was conducted at a single center (Toronto Western Hospital) Urology Department and a tertiary referral center in Ontario for SNM therapy, Toronto, Canada. We included all patients who were diagnosed with refractory $\mathrm{OAB}$, frequency-urgency syndrome, and chronic retention. Our selection criteria using urodynamic test (UDS) and voiding diaries included the following: refractory $\mathrm{OAB}$ with evidence of uninhibited bladder contraction on UDS, with no response to minimum of 2 types of anticholinergics and with diary evidence of a small amount per void less than $200 \mathrm{~mL}$; frequency, as duration $<4$ hours between voids during day time, with or without urge incontinence; frequency-urgency syndrome included evidence on voiding diary of a small amount per void less than $200 \mathrm{~mL}$, frequency $<4$ hours duration between voids during day time with or without urge incontinence and absence of uninhibited bladder contraction on UDS; chronic retention was confirmed on voiding diary with more than $30 \%$ of free voided urine evacuated by catheter. All patients were scheduled for stimulation test after completing a bladder diary for 3 consecutive days in order to quantify the degree of the lower urinary tract dysfunction. All patients underwent PNE test for 3 to 5 days as the first stimulation trial. Patients who encountered technical difficulty during PNE (i.e., patient who could not tolerate the procedure under local anesthesia), or had evidence of electrode migration underwent staged testing for 2 weeks. Patients with $\geq 50 \%$ improvement in subjective and/or objective symptoms recorded in their bladder diary completed during the test period (increased in calculated average volume per void, increased duration between voids and decreased voided volume by catheter) and maintained response after InterStim implantation are marked as True positive cases. No response to PNE test was marked as true negative after excluding electrode migration. In cases undergoing staged test trial, their results were compared to patient response after InterStim implantation.

\section{Statistical Analysis}

Data were collected using Microsoft Excel 2003. We used IBM SPSS Statistics ver. 19.0 (IBM Co., Armonk, NY, USA) and Statistics Calculator (Center for Evidence Based Medicine, Oxford, UK) for the diagnostic tests used in our analysis. Pearson correlation coefficient was calculated for sensitivity, specificity and patient characteristics (age, sex, and diagnosis). All values were considered statistically significant at $\mathrm{P} \leq 0.05$.

\section{Decision Algorithm}

All patients were scheduled for PNE screening test under local anesthesia in an outpatient setting. The sensory response specific for the anatomical site (anal, vaginal, scrotal, or perianal area) was determined in order to help localization of the electrode in the proper foramen. It was not necessary to evaluate the placement site by radiography. If any difficulty was encountered during PNE testing (i.e., the patient could not tolerate the 


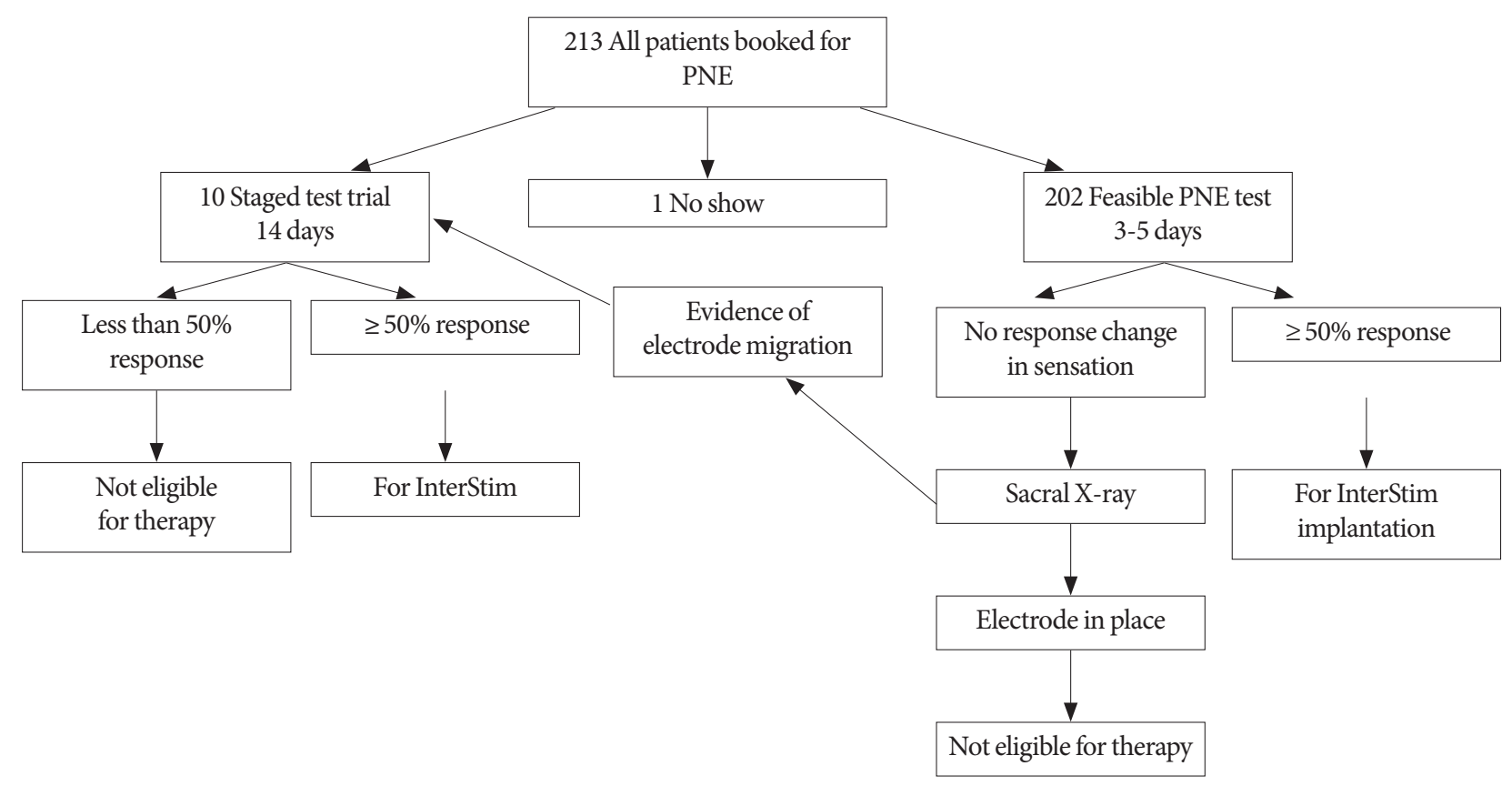

Fig. 1. Decision algorithm for selection of test trial. PNE, percutaneous nerve evaluation. InterStim (Medtronic Inc., Minneapolis, MN, USA).

procedure under local anesthesia), then the patient was selected for staged test trial.

For those who underwent PNE testing trial for 3 to 5 days, the response was assessed by comparing their bladder diaries. If a patient had $\geq 50 \%$ subjective improvement and/or objective response recorded in the bladder diary, then he/she was considered eligible for InterStim therapy. The implantation was performed in a single stage, under General anesthesia However, patients with the same level of sensation (in the perianal, scrotal, or vaginal areas), but with less than $50 \%$ positive response were not considered candidates for the therapy. For patients who demonstrated no response and presented no sensation at all, or for whom sensation diminished after a while, or location of sensation changed, additional sacral radiograms (anteriorposterior and lateral views) were obtained in order to diagnose electrode migration. If this was confirmed, the patients underwent staged procedure.

Staged test trial was performed in patients who had difficulties with PNE procedure (difficult foramen localization or patient could not tolerate the procedure) or radiological evidence of electrode migration. The procedure was performed in an operating room under general anesthesia, with appropriate prophylactic antibiotic. The tined lead electrode (3889, Medtronic Inc.) was inserted using fluoroscopic guidance and the location of the foramen (S3 or S4) was verified by an appropriate motor response of the levator ani muscles and the ipsilateral toe motion. Patients retained the tined lead for duration of 2 weeks. After this time, their response to stimulation was assessed as presented in Fig. 1.

\section{RESULTS}

A total of 213 patients, including $172(80.8 \%)$ female and 41 (19.2\%) male subjects were scheduled for PNE. Patients were diagnosed with refractory $\mathrm{OAB}(\mathrm{n}=102,47.9 \%)$, nonobstructive urinary retention $(n=63,29.6 \%)$, and frequency urgency syndrome ( $\mathrm{n}=47,22.1 \%)$. A total of $202(94.8 \%)$ had undergone PNE testing, 10 patients (4.7\%) underwent staged testing. Moreover, one patient declined PNE procedure, as presented in Table 1.

The overall sensitivity of PNE was $87.3 \%$ (95\% confidence interval [CI], 79.8-92.3) versus 90\% (95\% CI, 46.3-98.9) for staged test, and specificity for PNE was 98.5\% (95\% CI, 91.8-99.7) versus $92.9 \%$ (95\% CI, 56.1-99.2) for staged test. The positive predictive values and negative predictive values for PNE were 99\% (95\% CI, 94.4-99.8) and 82.1\% (95\% CI, 72.1-89.7) and those for staged test were 90\% (95\% CI, 46.3-98.9) and 92.9\% (95\% CI, 56.1-99.2), respectively. Positive likelihood ratio for PNE was 
Table 1. Patients demographic

\begin{tabular}{lc}
\hline Demographic & No. (\%) \\
\hline Total number of patients & 212 \\
Sex & \\
Female & $172(80.8)$ \\
Male & $41(19.2)$ \\
Test trial & \\
Percutaneous nerve evaluation & $202(94.8)$ \\
Staged & $10(4.7)$ \\
Diagnosis & \\
Refractory overactive bladder & $102(47.9)$ \\
Nonobstructive urinary retention & $63(29.6)$ \\
Frequency-urgency syndrome & $47(22.1)$ \\
\hline
\end{tabular}

One patient declined the procedure.

Table 2. Primary analysis of sensitivity and specificity for PNE test versus staged test trial

\begin{tabular}{lcc}
\hline Variable & PNE test trial & Staged test trial \\
\hline Sensitivity & $87.3 \%$ & $90.0 \%$ \\
Specificity & $98.5 \%$ & $92.9 \%$ \\
Positive predictive value & $99.0 \%$ & $90.0 \%$ \\
Negative predictive value & $82.1 \%$ & $92.9 \%$ \\
Positive likelihood ratio & 57.6 & 12.6 \\
\hline
\end{tabular}

PNE, percutaneous nerve evaluation.

Table 3. Pearson correlation between primary analysis result and patient's age, sex, and diagnosis

\begin{tabular}{lcc}
\hline Variable & Correlation coefficient & P-value \\
\hline Age & 10.77 & 0.056 \\
Sex & 9.68 & 0.085 \\
Diagnosis & 39.32 & 0.034 \\
\hline
\end{tabular}

57.6 compared to 12.6 in the staged procedure (Table 2).

Correlation analysis was performed for the patient's age, sex, and primary diagnosis. The only significant correlation was noticed for diagnosis (correlation, 39.32; $\mathrm{P}=0.034$ ) (Table 3). Secondary subgroup analysis was performed for the patient's diagnosis. In refractory $\mathrm{OAB}$ patients $\mathrm{PNE}$ test sensitivity and specificity were $89.2 \%$ and $98.1 \%$, compared to $83.3 \%$ and $75 \%$ for staged test trials, respectively. For patients with urine retention, staged test with $83.3 \%$ sensitivity was more sensitive than PNE having sensitivity of $79.2 \%$, which can be explained by the increased stimulation time required to elicit a response. In patients with frequency-urgency syndrome, results were similar to those in the OAB group, with $86 \%$ sensitivity for PNE test
Table 4. Sensitivity, specificity for PNE test versus staged test trial in refractory overactive bladder

\begin{tabular}{lcc}
\hline Variable & PNE test trial & Staged test trial \\
\hline Sensitivity & $89.2 \%$ & $83.3 \%$ \\
Specificity & $98.1 \%$ & $75 \%$ \\
Positive predictive value & $99.1 \%$ & $83 \%$ \\
Negative predictive value & $80.3 \%$ & $75 \%$ \\
Positive likelihood ratio & 48.4 & 3.33 \\
Negative likelihood ratio & 0.165 & 0.22 \\
\hline
\end{tabular}

PNE, percutaneous nerve evaluation.

Table 5. Sensitivity, specificity for PNE test versus staged test trial in chronic retention

\begin{tabular}{lcc}
\hline Chronic retention & PNE test trial & Staged test trial \\
\hline Sensitivity & $79.2 \%$ & $83.3 \%$ \\
Specificity & $95.8 \%$ & $83.3 \%$ \\
Positive predictive value & $95.0 \%$ & $83.3 \%$ \\
Negative predictive value & $82.1 \%$ & $83.3 \%$ \\
Positive likelihood ratio & 19 & 5 \\
Negative likelihood ratio & 0.217 & 0.2 \\
\hline
\end{tabular}

PNE, percutaneous nerve evaluation.

Table 6. Sensitivity, specificity for PNE test versus staged test trial in frequency- urgency syndrome, pelvic pain

\begin{tabular}{lcc}
\hline Variable & PNE test trial & Staged test trial \\
\hline Sensitivity & $86.0 \%$ & $50.0 \%$ \\
Specificity & $96.7 \%$ & $87.5 \%$ \\
Positive predictive value & $97.7 \%$ & $50.0 \%$ \\
Negative predictive value & $81.6 \%$ & $87.5 \%$ \\
Positive likelihood ratio & 27.52 & 4 \\
Negative likelihood ratio & 0.145 & 0.571 \\
\hline
\end{tabular}

PNE, percutaneous nerve evaluation.

compared to 50\% for staged test trials (Tables 4-6).

\section{DISCUSSION}

In the current study we used our algorithm for screening and selection of patients for the test. All patients underwent PNE test, which is simple as the first line screening test, while we limited the use of staged test procedure for special conditions (difficulty with foramen localization or evidence of electrode migration). We found that the proposed algorithm was feasible 
and applicable PNE when was used as first line screening test. It is also less expensive, as the tined leads (3889, Medtronic Inc.) are available at most medical centers. PNE test presents an acceptable price that makes it affordable as a screening test.

Electrode migration or displacement has been reported as the main reason for false negative results in PNE test $[9,10]$. This displacement can be detected by change in stimulation sensation pattern. This change was perceived either as a decrease or change in the sensation location. The electrode migration was confirmed by sacral radiography (anterioposterior images). In our study, we assessed patients' sensation (subjectively) and electrode location (objectively) to rule out electrode migration.

In our diagnostic calculations, we compared PNE and staged procedures with the primary diagnosis in the lower urinary tract dysfunction group. We found that these results were affected by the primary diagnosis (indication for SNM therapy). In refractory $\mathrm{OAB}$ and frequency-urgency group, $\mathrm{PNE}$ was considered both, a highly sensitive and specific test. However, in chronic nonobstructive retention, staged procedure was more sensitive than the PNE test. This difference could be explained by the increased stimulation time required to elicit a response that otherwise might be missed if the PNE alone is used [11].

Limitations of this study include the design. Adequate randomization with equally powered samples in each group might be the best design for such study. Among our study subjects, only 10 patients needed staged test in comparison to PNE group, without matching, but test sensitivity and specificity are usually not affected by the distribution of numbers when comparing two different tests.

In conclusion, the decision algorithm we used to screen patients for SNM therapy is a simple and reliable tool. PNE test has higher specificity and a positive predictive value in comparison to the staged procedure. We recommend PNE test, a simple office-based procedure, as the first line of screening in patients with lower urinary tract dysfunction prior to SNM therapy and to reserve staged test as a second-line alternative for screening.

\section{REFERENCES}

1. Hassouna MM, Siegel SW, Nÿeholt AA, Elhilali MM, van Kerrebroeck PE, Das AK, et al. Sacral neuromodulation in the treatment of urgency-frequency symptoms: a multicenter study on efficacy and safety. J Urol 2000;163:1849-54.

2. Abrams P, Andersson KE, Birder L, Brubaker L, Cardozo L, Chapple $\mathrm{C}$, et al. Fourth International Consultation on Incontinence Recommendations of the International Scientific Committee: Evaluation and treatment of urinary incontinence, pelvic organ prolapse, and fecal incontinence. Neurourol Urodyn 2010;29:213-40.

3. Shaker HS, Hassouna M. Sacral root neuromodulation in idiopathic nonobstructive chronic urinary retention. J Urol 1998;159:1476-8.

4. Al-Shaiji TF, Banakhar M, Hassouna MM. Pelvic electrical neuromodulation for the treatment of overactive bladder symptoms. Adv Urol 2011;2011:757454.

5. Banakhar MA, Al-Shaiji T, Hassouna M. Sacral neuromodulation and refractory overactive bladder: an emerging tool for an old problem. Ther Adv Urol 2012;4:179-85.

6. Williams ER, Siegel SW. Procedural techniques in sacral nerve modulation. Int Urogynecol J 2010;21 Suppl 2:S453-60.

7. Leong RK, De Wachter SG, Nieman FH, de Bie RA, van Kerrebroeck PE. PNE versus 1st stage tined lead procedure: a direct comparison to select the most sensitive test method to identify patients suitable for sacral neuromodulation therapy. Neurourol Urodyn 2011;30:1249-52.

8. Borawski KM, Foster RT, Webster GD, Amundsen CL. Predicting implantation with a neuromodulator using two different test stimulation techniques: a prospective randomized study in urge incontinent women. Neurourol Urodyn 2007;26:14-8.

9. Peters KM, Carey JM, Konstandt DB. Sacral neuromodulation for the treatment of refractory interstitial cystitis: outcomes based on technique. Int Urogynecol J Pelvic Floor Dysfunct 2003;14:223-8.

10. Spinelli M, Weil E, Ostardo E, Del Popolo G, Ruiz-Cerdá JL, Kiss G, et al. New tined lead electrode in sacral neuromodulation: experience from a multicentre European study. World J Urol 2005;23:2259.

11. Kessler TM, Madersbacher H, Kiss G. Prolonged sacral neuromodulation testing using permanent leads: a more reliable patient selection method? Eur Urol 2005;47:660-5. 